\title{
LA ALFABETIZACIÓN ECONÓMICA, HÁBITOS DE CONSUMO, ACTITUD HACIA EL ENDEUDAMIENTO Y SU RELACIÓN CON EL BIENESTAR PSICOLÓGICO EN FUNCIONARIOS PÚBLICOS DE LA CIUDAD DE PUNTA ARENAS
}

\author{
MARÍA G. HERRERA A.", CLAUDIA A. ESTRADA G." Y MARIANELA DENEGRI C."
}

\begin{abstract}
RESUMEN
El presente trabajo corresponde a una investigación trasversal correlacional que estudia relación que existiría entre variables económicas y el bienestar psicológico en una muestra de 100 funcionarios públicos de la ciudad de Punta Arenas. Instrumentos desarrollados y adaptados por investigadores chilenos permitieron la medición de la alfabetización económica, hábitos de consumo y actitud frente al endeudamiento así como del bienestar subjetivo. Los resultados indican que en términos de la alfabetización los participantes muestran niveles similares a los encontrados en otras investigaciones chilenas y que esta se vincula únicamente con el aspecto subjetivo del bienestar. Con relación a los hábitos de consumo, se aprecian buenos conocimientos a pesar que en términos del endeudamiento los niveles son altos. Se observó una relación entre la actitud austera de la escala de endeudamiento y la felicidad. Los resultados son discutidos en torno a su potencial aporte con respecto al desarrollo de programas preventivos de intervención psicosocial.
\end{abstract}

PALABRAS CLAVE: alfabetización económica, hábitos de consumo, bienestar psicológico.

\section{ECONOMIC LITERACY, CONSUMER BEHAVIOR, ATTITUDE TOWARS \\ DEBT AND ITS RELATION TO PSYCHOLOGICAL WELL BEING IN PUBLIC EMPLOYEES OF THE CITY OF PUNTA ARENAS}

\begin{abstract}
ASTRACT
The aim of this study is the association between economics variables and well-being in public employee from Punta Arenas city. Scales were developed and adapted by Chilean researcher. There were used to measure economic literacy, consumptions practices, attitude towards indebtedness and well-being. One hundred voluntaries participated in across-correlacional study. The results indicate that in terms of economic literacy level the sample has a similar median than others chilean' studies and this variable has association only with the subjective aspect of well-being. The consumptions practices are well
\end{abstract}

* Universidad de Magallanes, Av. Bulnes 01855, Punta Arenas. Chile.m_gladysherrera@hotmail.com, claudia.estrada@umag.cl.

* Universidad de la Frontera. Montevideo 0830, Temuco, Chile. marianela.denegricoria@gmail.com. 
known despite the high level of indebtedness. A correlation was observed between austere attitude and happiness. The results are discussed in relation with their contribution to the development of preventive programs for psychosocial intervention.

KEY WORDS: economic literacy, consumption practices, psychological wellbeing.

\section{INTRODUCCIÓN}

Una adecuada y equilibrada satisfacción de las necesidades vitales depende de un buen manejo de nuestros recursos, lo que redundará en una mejor adaptación social. De allí que ha aumentado el interés en la investigación del cómo las personas perciben y se relacionan con determinados fenómenos económicos así como el impacto de estos en su bienestar (ver Amar, Abello et al. 2007 Denegri y Palavecinos 2003; Denegri et al. 1999; Denegri et al. 2000; Luna et al. 2004 Ortega y Rodríguez 2004). Campo que recibe el nombre de psicología económica, disciplina que tiene como propósito el estudio de variables que permitan describir, explicar y predecir el comportamiento económico de los individuos y grupos sociales, así como explorar las formas en que los problemas económicos presentes en la sociedad afectan las conducta de la gente (Denegri 1998). De esta disciplina han surgido los conceptos de alfabetización económica, hábitos de consumo y actitud hacia el endeudamiento.

La alfabetización económica es un constructo que hace referencia a aquel conjunto de conceptos, habilidades, destrezas y actitudes que permiten al individuo la comprensión del entorno económico cercano y global, y la toma de decisiones eficientes de acuerdo a sus recursos financieros (Yamani, 1997). De acuerdo con Buckles y Melican (2002) un individuo económicamente alfabetizado es aquel que cuenta con las herramientas conceptuales y procedimentales mínimas para entender su mundo económico, interpretar los eventos que pueden afectarlo directa o indirectamente, y tomar decisiones personales y sociales sobre el sinfín de problemas económicos que se encuentran en la vida cotidiana. En un estudio sobre socialización económica en familias de clase media de la ciudad de Temuco, realizado por Denegri et al. (2005), se encontró que los padres no eran un vehículo adecuado para transmitir información de este tipo y que cada individuo debió adquirir conocimientos del mundo del dinero por sí mismo. Aunque los padres reconocen la importancia de la educación económica esto no se traduce en prácticas cotidianas de socialización.

Los hábitos o conductas de consumo han sido definidos como las actividades físicas, mentales y emocionales que las personas realizan cuando seleccionan o adquieren productos y servicios con el fin de satisfacer sus necesidades y deseos. La conducta del consumidor implicaría una serie de decisiones y actitudes sobre si consumir o ahorrar, sobre qué consumir, sobre la compra y el shopping, y sobre el uso de esos bienes (Robertson et al. 1984). Denegri et al. (1999) plantean la existencia de perfiles o estilos de conductas relacionadas con los hábitos de consumo: los denominados perfiles "reflexivo" e impulsivo. El primero referiría a un estilo de consumidor eficiente y eficaz, mientras que en el segundo predomina la "tendencia a conductas impulsivas de consumo", es decir, refiere a un consumidor con mayores posibilidades de efectuar compras poco eficaces vinculadas al uso del crédito como vía de acceso a los bienes. Denegri et al. (1999), plantean que estudios realizados en la Región de la Araucanía, indican la preponderancia del perfil "impulsivo", ocurriendo que un porcentaje importante de sujetos dice gastar más de lo que debería, lo que se asocia a sentimientos negativos y sensación de no ser capaz de cubrir todas sus necesidades, afectándose así la percepción subjetiva sobre la propia situación financiera.

La actitud frente al endeudamiento se define como la posición subjetiva que un individuo adopta respecto al uso del crédito para la satisfacción de sus necesidades materiales. Según Denegri et al. (1999) las actitudes hacia el endeudamiento mediarían en las decisiones de consumo. Habrían dos perfiles o estilos actitudinales: El "Austero" y el "Hedonista". El estilo actitudinal "austero", se caracteriza por un enfrentamiento cauteloso al endeudamiento, manteniendo una posición más tradicional en el manejo y administración de los recursos financieros. Este perfil evitaría el crédito y tendería al ahorro. El estilo actitudinal "hedonista", por su parte, se caracteriza 
por un uso del endeudamiento como recurso habitual para el logro de los bienes y servicios requeridos. Este perfil, entonces, utilizaría privilegiadamente las estrategias del crédito y no la del ahorro. Estudios recientes indican que un alto porcentaje de sujetos reconoce que el uso del crédito es parte esencial de su actual estilo de vida, pues le permite acceder a una batería de bienes mejorando su calidad de vida. Si bien es cierto las personas valoran la austeridad y se identifican con prácticas racionales en el uso del crédito, también reconocen que el crédito es una vía rápida de satisfacción de la necesidad de consumo. Denegri et al. (1999) destaca el hecho de que el uso masivo del crédito ha cambiado el perfil de la sociedad transformándola en sociedades de consumo. Wilkie (1994) señala que los símbolos de estatus son productos que sirven para enviar a otros mensajes sobre el estatus social elevado de una persona; también sirven a menudo para decirle a la propia persona internamente que él/ella ha llegado o lo ha logrado, o tiene un valor personal (Luna et al. 2004) plantean que se hace necesario promover acciones educativas desde temprana edad lo que nos permitirá una mejor comprensión de por qué compramos y como ello se relaciona con nuestra identidad.

El concepto de bienestar humano es considerado un elemento fundamental de la "salud mental" (OMS, 2009) y ha sido ampliamente estudiado. Aún no existe completo consenso respecto de sus componentes, podría definirse como un estado de satisfacción personal con la vida que experimentan las personas, lo que tiene su punto de partida en la transacción entre el individuo y su entorno micro y macro social, con sus elementos actuales $e$ históricos, donde se incluyen condiciones objetivas materiales y sociales que le brindan determinadas oportunidades para su realización personal (García y Gonzalez, 2000). Para la OMS (2009) el bienestar incluye estar consciente de las propias capacidades, ser capaz de afrontar las tensiones normales de la vida, trabajar de forma productiva y contribuir positivamente a la sociedad. Los diferentes modelos teóricos concuerdan en dividirlos en dos componentes: los objetivos - condiciones de vida - y los subjetivos - satisfacción personal - (Borthwick-Duffy, 1992). Los componentes objetivos han sido descritos en el Índice de Desarrollo Humano (IDH), establecido por las Naciones Unidas para medir el grado de desarrollo de los países a través del Programa para el Desarrollo (PNUD) e incluye, como variables principales la esperanza de vida, la educación y el producto interior bruto. Los componentes de la dimensión subjetiva han sido ampliamente discutidos y se han considerado variables tales como la felicidad (Argyle 1992; Fierro 2000), la satisfacción con la vida (Veenhoven, 1994) y la presencia de afectos positivos o negativos, (Bradburn, 1996). El bienestar sería el nivel de satisfacción global que las personas poseen en virtud de sus oportunidades vitales (recursos sociales, personales y aptitudes individuales), los acontecimientos que enfrenta (privación- opulencia, ataque, protección, soledad, compañía etc.) y la experiencia personal que resulta de todo lo anterior. En otros términos, serían el grado o nivel de agrado que una persona manifiesta respecto de su propia vida (Veenhoven, 1994). Autores como Ryff (1989) sugieren un modelo multidimensional de Bienestar Psicológico, el que se encontraría constituido por cinco dimensiones: (i) control de situaciones, (ii) aceptación de sí mismo, (iii) vínculos psicosociales, (iv) autonomía y (v) proyectos personales. El control de situaciones es una medida de funcionamiento psicológico eficiente que permite evaluar el manejo efectivo del medio ambiente y de la propia vida. Se manifiesta en el sujeto por medio de la habilidad para producir cambios en su entorno asegurando, de esta forma la satisfacción de sus necesidades. Las personas con mejor control de situaciones tienen una sensación de autoeficacia más elevada y por lo mismo son capaces de tolerar mejor sus fracasos. La Aceptación de sí mismo por otra parte, implica la conformidad de los múltiples aspectos del sí mismo, así como una apreciación positiva de estos. Una persona con una alta aceptación de sí mismo, muestra conformidad con su cuerpo, con su forma de ser, su pasado, y es capaz de aceptar sus equivocaciones buscando mejorar (Castro et al., 2002). La dimensión de vínculos psicosociales refiere a la capacidad para construir relaciones de confianza con otras personas, y mostrar empatía y afectividad. Además, los vínculos psicosociales incluirían las redes de apoyo disponibles frente a situaciones de dificultad (Castro et al., 2002). La Autonomía es la capacidad de tomar decisiones de forma independiente, resistiéndose a la influencia grupal, expresando lo que se piensa y autorregulando el propio comportamiento. Una persona autónoma 
sería capaz de regirse por sus propias reglas y ser independiente de la influencia externa. La dimensión proyectos personales sería la creencia de que la vida tiene un propósito y significado, y que estos le dan sentido y estructura a nuestra cotidianeidad (Barrientos, 2006). Un adecuado desarrollo en esta dimensión tendría como consecuencia un bienestar asociado al sentimiento de propositividad existencial. Finalmente, otros autores incluyen la dimensión de felicidad, lo que alude a un estado psicológico emocional de satisfacción vital (Arita et al., 2005)

Estas dimensiones del bienestar pueden ser evaluadas en diferentes contextos, los que han sido denominados dominios de satisfacción. Estos dominios serían diversas áreas de la vida adulta en las que el bienestar puede presentarse de manera diferencial. Estos serían: (i) el laboral, (ii) el de relaciones interpersonales, (iii) el material y (iv) el subjetivo (Sánchez-Canovas, 1998). El dominio laboral sería el ambiente y las actividades en las que se desarrollan actividades de trabajo y/o estudio; el de relaciones interpersonales abarcaría los vínculos privados de amistad, familia y relaciones amorosas; el material está constituido por los recursos económicos y los bienes de los que se dispone, y el subjetivo está conformado por el mundo interno y las vivencias subjetivas de cada individuo.

La relación entre las variables económicas y el bienestar subjetivo ha sido escasamente estudiada. El Bienestar Psicológico se ha vinculado con una serie de categorías sociales, las que por un lado tienen que ver con las condiciones materiales de vida, los estilos de vida que adopta una determinada población, el desarrollo económico, siendo este último concepto el que ha sido más ligado a un adecuado nivel de vida, noción que se relaciona directamente con indicadores como el ingreso y el consumo.

Son pocos los estudios que aportan antecedentes sobre las formas en que los adultos comprenden el mundo económico, a pesar de que en esta etapa de la vida nos integramos al mundo laboral y por lo tanto nos hacemos cargo del manejo de nuestras finanzas personales (Stacey 1987; Burgoyne et al. 1997; Webley, 1999). Aumentar el conocimiento en esta área reviste gran importancia dado que del adecuado manejo de los recursos depende la satisfacción de las necesidades vitales. Pérez (2006), establece que un inadecuado uso de los recursos financieros produce desestabilización personal y familiar, inseguridad laboral, debilitación de los vínculos sociales y estrés entre otros efectos. Stingler (1971) en tanto postula la importancia de la comprensión de la economía para el logro de una mejor calidad de vida ya que el manejo eficiente de información económica actúa como una herramienta comunicacional entre las personas facilitando la interpretación de los eventos que les afectan en este campo. Esta comprensión del mundo económico ayudará al individuo a hacer frente a las exigencias de un mundo globalizado que plantea nuevos escenarios económicos.

El objetivo de la presente investigación fue abordar la relación existente entre las variables nivel de alfabetización económica, los hábitos de consumo y la actitud hacia el endeudamiento, y el bienestar psicológico en funcionarios públicos de la ciudad de Punta Arenas. Nuestra hipótesis de investigación plantea que tales variables se asocian en forma significativa.

\section{MÉTODO}

\section{Participantes y diseño}

Participaron 100 funcionarios públicos de la ciudad de Punta Arenas quienes fueron seleccionados mediante muestreo no aleatorio intencional. El 99\% fue de género masculino y la moda de edad se situó en el rango 45-64 años. Según estado civil la mayoría está casado (56\%) y le siguen los solteros (38\%). Se utilizó un diseño no experimental transversal correlacional.

\section{Instrumentos}

Los instrumentos fueron seleccionados por su amplio uso en investigaciones previas en población chilena lo que favorece su validez y confiabilidad (ver referencias bibliográficas para más información sobre cada uno de ellos).

Test de Alfabetización económica para adultos TAE-A, Gempp et al., (2007), Consta de 23 preguntas las cuales miden el nivel de comprensión de conceptos y prácticas necesarias para un desempeño económico eficiente. Evalúa los siguientes conceptos económicos: economía general, microeconomía, macroeconomía y economía internacional. Para la presente muestra alcanzó un nivel de confiabilidad de 0,6 (Alfa). 
Cuestionario de bienestar Psicológico, Estrada y Avendaño, (2009): Cuestionario que mide el nivel de bienestar psicológico que las personas experimentan respecto de sus vidas. Fue construido integrando dos modelos distintos de bienestar; dominios de bienestar: subjetivo, laboral, relaciones interpersonales y material; y dimensiones de bienestar: control de situaciones, aceptación de sí mismo, vínculos psicosociales, proyectos, autonomía y felicidad. El instrumento compuesto por 44 ítems, en el que cada afirmación mide un dominio y una dimensión simultáneamente que se responden en base a una escala Likert de 5 puntos que va desde el 1:"Totalmente en desacuerdo" hasta el 5:"Totalmente de acuerdo". En su construcción se utilizó criterio inter-jueces para determinar su validez de contenido y análisis factorial para la validez de constructo. Para la presente muestra alcanzó un nivel de confiabilidad de 0,9 (Alfa). Se cuenta con normas locales percentilares, según género, realizadas sobre una muestra de 840 habitantes de Punta Arenas.

Escalas de actitud hacia el endeudamiento (Denegri y col., 1999): Permite evaluar la actitud de las personas frente las deudas que posee como individuo. Posee dos subescalas una que mide estilo actitudinal austero (Propensión hacia el uso cauto del dinero, orientación hacia el ahorro y evitación del crédito) y otra que mide estilo actitudinal hedonista (Tendencia hacia uso del dinero como medio para el logro de satisfacciones, orientación hacia el uso de crédito y al endeudamiento). Para la presente muestra alcanzó un nivel de confiabilidad de 0,6 (Alfa).

Escala de hábitos y conductas de consumo: (Denegri y Cools, 1997): Mide la organización de la conducta de compra del consumir en vía a discriminar entre actos de consumo racionales, planificados y autocontrolados, y actos de consumo impulsivos. Posee dos subescalas, la denominada reflexiva (consumidor eficiente y eficaz) y la impulsiva (consumidor poco eficaz). Para la presente muestra alcanzó un nivel de confiabilidad de 0,65 (Alfa).

\section{Procedimiento}

Cada participante contestó los instrumentos de forma individual. Fueron contactados en su lugar de trabajo y luego de exponérseles los objetivos centrales del estudio firmaron un consentimiento informado. La aplicación se hizo en una única sesión.
Luego de finalizar se les agradeció su participación y se les indicó el procedimiento para obtener sus resultados personales.

\section{RESULTADOS}

Los resultados generales indican que un $81 \%$ de los participantes alcanza un nivel de alfabetización económica que los sitúa en el nivel medio $(M=9,8$; $D E=2,2$ ). En otros términos, la mayoría cuenta con nociones básicas sobre aspectos generales del funcionamiento de la economía que tienen relación con el gasto, ahorro, oferta, demanda y el uso del dinero.

La evaluación de los hábitos y conductas de consumo muestra que un $82 \%$ alcanzan puntajes intermedios en esta variable mientras que un 13\% mostró puntajes altos y el 5\% restante puntajes bajos. Los resultados de las sub-escalas que permite discriminar entre actos de consumo planificados y autocontrolados (perfil reflexivo), versus actos impulsivos, indican que el promedio de conductas reflexivas $\left(M=16,2\right.$, pto. Neutro $=14, t_{(100)}=6,54$, $p<0,001)$ se sitúa sobre el punto medio de la escala mientras que las conductas impulsivas $(M=4,08$, pto. Neutro $\left.=4, t_{(100)}=0,47, n s\right)$ se sitúan en este punto neutro. En otros términos, los participantes muestran un promedio alto en comportamientos en consumo reflexivo e intermedio en consumo impulsivo.

Respecto al nivel de endeudamiento de la población estudiada, los resultados indican que en un $72 \%$ los encuestados refieren tener un alto nivel, mientras que el 28\% restante se sitúa en un nivel medio. En otros términos una alta proporción de los encuestados percibe tener un alto endeudamiento. En las subescalas de actitud frente al endeudamiento, aquella que evalúa actitud austera muestra un promedio significativamente por sobre el punto neutro de la escala $\left(M=32,17\right.$, pto. Neutro $=14, t_{(100)}=$ $61,36, p<0,001)$, al igual que el promedio de actitud hedonista $\left(M=10,72\right.$, pto. Neutro $=8, t_{(100)}=7,37$, $p<0,001)$. Los participantes muestran una actitud hacia el endeudamiento que es al mismo tiempo alta en austeridad y alta en hedonismo.

Las variables económicas muestran algunas asociaciones entre sí. El estilo de endeudamiento austero se relaciona negativamente de forma marginal con la alfabetización económica $\left(r_{(100)}=-0,15\right.$, 


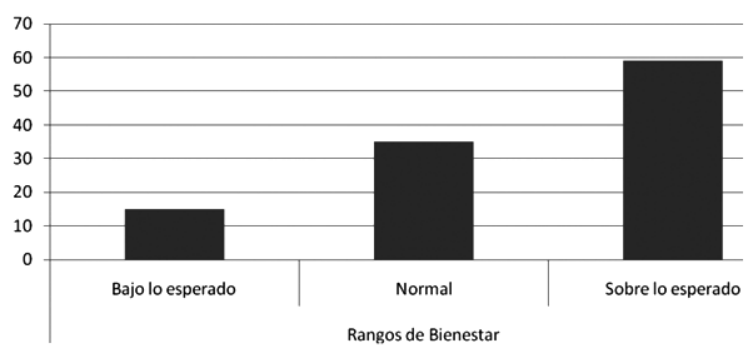

Fig. 1. Porcentaje de participantes según rango de pertenencia.

$p=0.08)$, de forma negativa y significativa con el endeudamiento hedonista $\left(r_{(100)}=-0,27, p=0,003\right)$ y los hábitos de consumo impulsivos $\left(r_{(100)}=-0,36\right.$, $p=0,001$ ).

Los hábitos de consumo correlacionan negativamente con el endeudamiento austero $\left(r_{(100)}=\right.$ -0,28, $p=0.04$ ) indicando que puntajes altos en hábitos de consumo se acompañan de bajos puntajes en endeudamiento.

La medición del Bienestar subjetivo por su parte indica que el 50\% se sitúa dentro del rango de bienestar sobre lo esperado, un 35\% se sitúa dentro de la zona de normalidad y el 15\% restante bajo lo esperado (ver figura 1). El promedio global de bienestar se sitúa por sobre el punto medio de la escala $\left(M=4,5\right.$; pto. Neutro $\left.=3, t_{(100)}=37,4, p<0,0001\right)$.

$\mathrm{Al}$ analizar las relaciones existentes entre las variables económicas del estudio, alfabetización económica, hábitos de consumo y actitud hacia en endeudamiento, con el bienestar psicológico, se observa que no existen asociaciones significativas entre ellas. Ni la alfabetización económica $\left(r_{(100)}=\right.$ 0,06 , n.s.), ni los hábitos de consumo $\left(r_{(100)}=0,02\right.$, n.s.), ni la actitud hacia el endeudamiento $\left(r_{(100)}=\right.$ 0,08 , n.s.), muestra asociaciones con el bienestar total. Al asociar las sub-escalas de actitud hacia el endeudamiento con el bienestar subjetivo global, se observa que existe una asociación significativa entre ésta y el estilo de endeudamiento austero $\left(r_{(100)}=\right.$ $0,20, p<0,02$ ), indicando que existe mayor bienestar entre las personas con mayores puntajes en estilo austero y viceversa. No se observa asociación entre el estilo de endeudamiento hedonista y bienestar psicológico global, $r_{(100)}=0,04$, n.s.

La relación entre las diferentes áreas del bienestar subjetivo: material, subjetivo, afectivo y laboral, y las variables económicas, indican únicamente una relación positiva entre endeudamiento total y el área subjetiva del bienestar $\left(r_{(100)}=.19, p=0.03\right)$.

Al correlacionar las dimensiones del Bienestar Subjetivo (Control de situaciones, aceptación de sí mismo, vínculos psicosociales, autonomía, proyectos y felicidad) con las variables económicas totales, no se observan correlaciones significativas. Al asociar las subescalas de estas variables encontramos una relación positiva entre la actitud austera de endeudamiento y felicidad $\left(r_{(100)}=.23, p=0.01\right)$ y entre este estilo de endeudamiento y la dimensión de autonomía $\left(r_{(100)}=.19, p=0.04\right)$. El aumento de una actitud austera se acompaña de un aumento de la felicidad y viceversa; lo mismo ocurre con esta actitud y la autonomía entendida como la capacidad de ser independiente en sus decisiones.

Al cruzar las áreas y dimensiones del bienestar encontramos que el perfil hedonista presenta asociaciones negativas con el área laboral en sus dimensiones control de situaciones, $\left(r_{(100)}=.18, p=\right.$ $0.04)$, proyectos $\left(r_{(100)}=.19, p=0.03\right)$, felicidad $\left(r_{(100)}\right.$ $=.17, p=0.05)$, y en el área material en su dimensión de felicidad $\left(r_{(100)}=.20, p=0.02\right)$. (ver tabla $\left.\mathrm{N}^{\circ} 1\right)$

En el mismo análisis respecto del perfil austero, éste muestra asociaciones positivas con el área laboral en su dimensión de aceptación de si mismo $\left(r_{(100)}=\right.$ $.18, p=0.04)$, el área afectiva de relaciones interpersonales en sus dimensiones, vínculos psicosocial $\left(r_{(100)}=.20, p=0.02\right)$, autonomía $\left(r_{(100)}=.23, p=\right.$ $0.01)$ y felicidad, $\left(r_{(100)}=.26, p=0.005\right)$. En otros términos, el aumento en austeridad se acompaña principalmente de bienestar en el área interpersonal mientras que el aumento de un perfil hedonista se acompaña principalmente disminución del bienestar en el área laboral (Ver tabla $\mathrm{N}^{\circ} 2$ ).

Tabla 1. Correlaciones entre el cruce de áreas y dimensiones Bienestar y la actitud hedonista.

\begin{tabular}{llcc}
\hline & & Fuerza y dirección & Significación \\
\hline 1. & Laboral-Control de situaciones & -0.18 & 0.04 \\
2. & Laboral- Proyectos & -0.19 & 0.03 \\
3. & Laboral-Felicidad & -0.17 & 0.05 \\
4. & Material-Felicidad & -0.20 & 0.02 \\
\hline
\end{tabular}


Tabla 2. Correlaciones entre el cruce de áreas y dimensiones Bienestar y la actitud austera.

\begin{tabular}{llcc}
\hline & & Fuerza y dirección & Significación \\
\hline 1. Laboral-acept. de si mismo & 0.12 & 0.04 \\
2. Interpersonal- Vínculos & 0.20 & 0.02 \\
3. Interpersonal-Autonomía & 0.23 & 0.01 \\
4. Interpersonal-Felicidad & 0.26 & 0.001 \\
\hline
\end{tabular}

\section{DISCUSIÓN Y CONCLUSIONES}

El objetivo de la presente investigación consistió en escrutar las posibles asociaciones entre las variables constituidas por factores de orden económico y factores de orden psicológico, reunidos bajo el concepto de bienestar subjetivo. Los resultados indican que esta relación se presente en forma limitada, siendo la más significativa aquella constatada entre el endeudamiento total y el bienestar, en el sentido de que mientras más cauta es la actitud de los individuos frente al endeudamiento también mayor es el bienestar que experimentan. Este resultado se ve reafirmado al considerarse la vinculación entre los perfiles "austero" y "hedonista" (que componente la variable endeudamiento), por cuanto la austeridad se acompaña de un mayor bienestar, y el incremento del hedonismo se acompaña de una disminución del mismo. Estos resultados son coherentes con respecto a lo que teóricamente entendemos como bienestar (la capacidad de estar consciente de las propias capacidades y afrontar correctamente las tensiones de la vida), por cuanto el perfil austero se caracteriza por la eficiencia en el manejo de los recursos económicos, siendo, por lo tanto, un estilo realista en cuanto al desarrollo de las modalidades de satisfacción de las necesidades.

Son también relevantes las asociaciones entre los perfiles de endeudamiento y los cruces de áreas y dimensiones del bienestar que indican que los puntajes altos en el perfil "austero" se relacionan con puntajes altos en tres aspectos de bienestar vinculados con las relaciones interpersonales, mientras que los puntajes altos en el perfil "hedonista" se asocian a puntajes bajos en tres aspectos del bienestar vinculado con las relaciones laborales. Se podría esgrimir, que un manejo cauteloso de los recursos financieros tiene efectos favorables en algunas áreas de las relaciones interpersonales probablemente porque este estilo de enfrentamiento del endeudamiento sería menos estresante en la vida cotidiana, favoreciendo los vínculos, la sensación de autonomía y la felicidad.
Por otro lado, hipotéticamente, el estilo hedonista podría tener impacto en el área laboral ya que el uso del crédito como forma de obtener bienes y servicios podría provocar tensiones, disminuyendo la sensación de controlar las situaciones, afectando negativamente el desarrollo de proyectos, y mermando el sentimiento de felicidad. Este estudio correlacional, no permite responder a estas cuestiones ya que no establece relaciones de causalidad, pero si abre el debate respecto al efecto diferencial que estos estilos de endeudamiento pudiesen tener sobre algunas áreas del bienestar.

La alfabetización económica promedio se situó en el nivel intermedio, resultado que sitúa a la muestra estudiada dentro de lo esperado en comparación con los resultados obtenidos por otros estudios realizados en nuestro país, que indican que generalmente los sujetos poseen ciertas habilidades para realizar un adecuado manejo de sus finanzas personales, aunque no las suficientes para una adecuada comprensión del fenómeno económico.

Los resultados de la variable hábitos de consumo resultan particularmente interesantes ya que muestran simultáneamente alta adherencia a un perfil reflexivo de consumo e intermedia adhesión a un perfil impulsivo. Dado que ambos son perfiles incompatibles y correlacionan negativamente, es posible suponer, que el resultado observado para el perfil reflexivo responde -parcialmente al menos- a la presencia de alta deseabilidad social. Otro argumento que apoya este planteamiento es el alto nivel de bienestar psicológico general observado en la muestra, la que alcanza niveles por sobre lo esperado y por encima de lo observado en muestras de otras investigaciones. Ninguno de los instrumentos mencionados controla dicha variable y las características laborales de la muestra (empleados públicos), puede haber afectado estas mediciones. El marco conceptual permitía predecir una relación entre las actitudes hacia el endeudamiento y los hábitos de consumo así como de estas con la alfabetización económica. La necesidad de 
aprobación social y aceptación, y la creencia de que ésta se puede obtener por medio de conductas culturalmente aceptables y deseables, así como la percepción de que se está siendo evaluado en su adecuación social, pueden impulsar a las personas a optar por respuestas que considera socialmente apropiadas (Marlowe y Crowne, 1961).

A pesar de que los resultados generales son modestos y muestran correlaciones bajas entre algunas de las variables estudiadas, nos entregan importantes pistas de análisis y de intervención; en primer lugar, aunque no forma parte de las variables de estudio, los participantes muestran un nivel objetivo de alto endeudamiento, lo que, aunque contradice algunas de las medidas observadas, afirma algunas otras. El nivel de alfabetización económica, los puntajes de actitud frente al endeudamiento relacionados con la impulsividad y los hábitos de consumo hedonistas son compatibles con tal hecho (Un 72\% de participantes que declaran un alto endeudamiento). Estos elementos permitirán concebir estrategias de intervención psicosocial que prevengan problemas de salud mental asociados con esta situación.

En futuras investigaciones sobre esta temática se impone la inclusión de una escala de deseabilidad social para corregir el efecto de esta variable. La decisión de realizar el presente estudio en una muestra de funcionarios públicos fue fundamental para comprender el efecto, de que sentirse evaluado y potencialmente "juzgado", tiene en la medición de variables tan sensibles como las de orden económico. No obstante, lo anterior no invalida el resultado principal de esta investigación que indica que el bienestar es acompañado de un estilo austero de hábitos de consumo. La planificación de intervenciones para esta problemática deberá considerar tanto el peso de la deseabilidad social como del peso de la presión social hacia el consumo; se hace necesario que las creencias de que poseer bienes acompaña a la felicidad sean moderadas por el reconocimiento de las consecuencias que el sobreendeudamiento tiene sobre el bienestar de las personas.

\section{AGRADECIMIENTOS}

Agradecemos la valiosa colaboración del profesor Sergio Avendaño quien contribuyó de forma importante a la claridad de este texto.

\section{BIBLIOGRAFÍA}

AMAR, J., R. ABELLO, M. DENEGRI, M. LLANOS y R. SUÁREZ 2007. Estrategias de socialización económica en padres de familia. Revista de economía del Caribe. 1:156-183.

ARDILA, R. 2003. Calidad de vida: una definición integradora. Revista Latinoamericana de Psicología. 35(2):161-164.

ARITA, B. 2005. La capacidad y el bienestar subjetivo como dimensiones de estudio de la calidad de vida. Revista colombiana de Psicología, 1:73-79.

ARGYLE, M. 1992. The Social Psychology of Everyday Life. Routledge

ARITA, B., S. ROMANO, N. GARCÍA y M. FÉLIX 2005. Indicadores objetivos y subjetivos de la calidad de vida. Enseñanza e Investigación en Psicología. 10(1):93-102.

BARRIENTOS, J. 2006. Calidad de vida, Bienestar subjetivo: Una Mirada psicosocial. Universidad Diego Portales, Santiago.

BRADBURN, N. 1969. The structure of psychological wellbeing. Aldine, Chicago.

BORTHWICK-DUFFY, S.A. 1992. Quality of life and quality of care in mental retardation. En: Mental retardation in the year 2000, Editor Rowitz L., (pp.52-66), SpringerVerlag, Berlin.

BUCKLES, S. y C. MELICAN 2002. Assessment framework: 2006 National Assessment of Educational Progress in Economics. National Assessment Governing Board, Washington.

BURGOYNE, C., S. LEA, P. WEBLEY y B. YOUNG 1997. Introductory notes for the session on becoming an economic adult. Department of Psychology. University of Exeter, United Kingdom.

CASTRO, A., A. BRIZZIO y M. CASULLO 2002. Factores salugénicos en adolescentes argentinos. Un estudio preliminar con la escala de sentido de coherencia de Antonovsky. Investigaciones en Psicología. año 8 (1) Facultad de Psicología. U.B.A. ISSN: 0329-5893. pp: 55-77

DENEGRI, M. 1998. La construcción de nociones económicas en la infancia y la adolescencia. En: Desarrollo humano, perspectivas siglo XXI. Editores J. Ferro y J. Amar, Uninorte, Colombia.

DENEGRI, M. 2004. Introducción a la Psicología Económica. Psicom Editores. Bogotá.

DENEGRI, M., J. DELVAL, M. PALAVECINOS, M. KELLER y A. GEMPP 2000. Desarrollo del pensamiento económico en la infancia y la adolescencia. Boletín de Investigación Educacional, 13:291-308. 
DENEGRI, M., M. FERNANDEZ, R. ITURRA, M. PALAVECINOS y M. RIPOLL 1999. Consumir para vivir y no vivir para consumir. Ediciones Universidad de la Frontera. Temuco.

DENEGRI, M., R. GEMPP, C. DEL VALLE, S. ETCHEBARNE y Y. GONZÁLEZ 2006. El aporte de la psicología educacional a las propuestas de educación económica: los temas claves. Revista de psicología, 15 (2):77-94.

DENEGRI, M., G. MARTÍNEZ y S. ETCHEBARNE 2007. La comprensión del funcionamiento bancario en adolescentes chilenos: un estudio de Psicología Económica. Interdisciplinaria, 24(2):137-159.

DENEGRI, M., y M. PALAVECINO 2003. Género y alfabetización económica ¿Oportunidades de desarrollo o nuevos caminos para la discriminación? Psicología del Caribe, 12:76-97.

DENEGRI, M., C. DEL VALLE, R. GEMPP y M. LARA 2006. Educación económica en la escuela: Hacia una propuesta de intervención. Estudios Pedagógicos, 32(02):103-120.

DENEGRI, M., M. PALAVECINO Y R. GEMPP 2005. Socialización económica en familias Chilenas de clase media, educando ciudadanos o consumidores. Psicología y sociedades. 17 (2):54-68.

DENEGRI, M., M. PALAVECINO y M. RIPOLL 1998. Consumir para vivir y no vivir para consumir. Ediciones Universidad de la Frontera, Temuco.

DÍAZ, D., y A. BLANCO 2005. El Bienestar su concepto y medición. Psicothema. 17(4):582-589.

FIERRO, A. 2000. Sobre la vida feliz. Ediciones Aljibe, 200 pp. (1982), Málaga.

GARCÍA, C. y A. GONZÁLEZ 2000. La categoría Bienestar Psicológico. Su relación con otras categorías sociales. Revista Cubana Medicina General Integral. 16(6):586-92.

GEMPP, R., M. DENEGRI, N. CARIPÁN, V. CATALÁN, S. HERMOSILLA y C. CAPRILE 2007. Desarrollo del Test de Alfabetización Económica para Adultos TAE-A-25. Interamerican Journal of Psychology, 41(3):275-284.

LUNA, R., S.H. PUELLO y M. BOTERO 2004. La compra impulsiva y el materialismo en los jóvenes: Estudio exploratorio en estudiantes Universitarios de Barranquilla. Psicología desde el Caribe. 14:1-26.
MARLOWE, C. y D. CROWNE 1961. A new scale of social desirability independent of psychopathology. Journal of Consulting Psychology, 24:349-354

OMS (2009): La manera de hacer las cosas. Programa de las Naciones Unidades para el Desarrollo Humano Santiago: PNDU

ORTEGA, V. y J.C. RODRÍGUEZ-VARGAS 2004. Escala de Hábitos y conductas de consumo: Evidencias sobre dimensionalidad. International Journal of Clinical and Heath Psychology, 4:121-136

PÉREZ, L. 2006. Seminario, Porqué se sobreendeudan los Chilenos y Chilenas? Acercamiento para la comprensión de la problemática del sobreendeudamiento en Chile. Consumers International Red Puentes-Chile. Universidad Alberto Hurtado. pp. 8

SÁNCHEZ-CANOVAS, J. 1998. EBP: Escala de Bienestar psicológico. TEA ediciones. Madrid.

STACEY, B. 1987. Economic socialization. En: Annual Review of Political Science, Editor S. land, 2:1-33.

STINGLER, G. 1971. The theory of economic regulation. Bell Journal of Economics and Management Science, 2:3-21

ROBERTSON, T.S., J.Z. ZIELINSKI y S. WARD 1984. Consumer Behavior. Scott: Foresman and Company

RYFF, C. 1989. Happiness is everythong, or is it? Explorations on the meaning of psychological well-being. Journal of Personality and Social Psychology, 57:1069-1081.

VEENHOVEN, R. 1994. Is happiness a trait? Test of the theory that a better society does not make people any happier. Social Indicators Research, 32:101-160.

WEBLEY, P. 1999. The economic psychology of everyday life: Becoming an economic adult. Documento de trabajo. Universidad de Exeter, Inglaterra.

WILKIE, W. 1994. Consumer Behavior (3era Edición), John Wile \& Sons, Inc. New York.

YAMANI, E. 1997. The meaning of "economics educations" in Japanese elementary and secondary education: An historical perspective. En: Proceeding of the second conference of the international association for children's social and economic education. Malmö Suiden: Edge hill university college/IACSEE. p. 101. 
